\section{OC-064 HEPATIC FIBROSIS IN PEOPLE WITH TYPE 2 DIABETES MELLITUS: THE EDINBURGH TYPE 2 DIABETES STUDY}

doi:10.1136/gutjnl-2012-302514a.64

1J R Morling,* ${ }^{2} \mathrm{M}$ W Strachan, ${ }^{2} \mathrm{R}$ M Williamson, 'J F Price, ${ }^{3} \mathrm{~J}$ A Fallowfield, ${ }^{4}$ I N Guha. ${ }^{1}$ Centre for Population Health Sciences, University of Edinburgh, Edinburgh, UK; ${ }^{2}$ Metabolic Unit, Western General Hospital, Edinburgh, UK; ${ }^{3}$ MRC Centre for Inflammation Research, University of Edinbugh, Edinburgh, UK; ${ }^{4}$ Nottingham Digestive Disease Centre BRU, University of Nottingham, Nottingham, UK

Introduction People with type 2 diabetes mellitus (T2DM) have a higher prevalence of and higher mortality rates from liver-associated disease than the non-diabetic population of equivalent age. Early identification of liver disease followed by appropriate surveillance and intervention should be beneficial to the expanding population of older people with T2DM. We aimed to determine the prevalence and progression of hepatic fibrosis in a representative sample of older adults with T2DM

Methods 1066 participants of the Edinburgh Type 2 Diabetes Study, a large, randomly-selected population of patients with T2DM aged 60-75 years were invited to assessment on two occasions. At baseline, the Enhanced Liver Fibrosis Panel (ELF) was measured and demographics and diabetes history were recorded. ELF was repeated approximately 3 years later. The presence of fibrosis was determined as: none/mild $<7.7$, moderate $7.7-<9.8$, severe $\geq 9.8$. Fibrosis progression was investigated for association with demographics, diabetes history and alcohol intake using logistic regression.

Results Average follow-up was 2.7 years. At baseline $(n=530), 3.1 \%$ subjects had no/mild fibrosis, $85.1 \%$ moderate fibrosis and $11.9 \%$ severe fibrosis. Follow-up prevalences $(n=806)$ were $1.9 \%, 79.3 \%$, and $18.9 \%$ respectively. 489 subjects had both baseline and followup ELF results. 61 (12.5\%) patients progressed to the most severe fibrosis stage between baseline and follow-up. None of the factors investigated were independently associated with progression to severe fibrosis: age, sex, social deprivation, diabetes-related factors (fasting glucose, HbA1c, diabetes duration, treatment type or location) or alcohol use.

Conclusion These preliminary data suggest that considerable hepatic fibrosis is present in older patients with T2DM. ELF is well validated for diagnosing severe hepatic fibrosis and it is of concern that in $<3$ years, almost $13 \%$ of patients progressed to this level. Further work is planned to determine factors affecting progression and to develop prognostic models for the development of hepatic fibrosis in people with T2DM.

Competing interests None declared.

\section{OC-065 TARGETED INHIBITION OF TISSUE FACTOR AND THROMBIN ON CD31 EXPRESSING CELLS SUPPRESSES HEPATIC FIBROSIS IN CCL4 TREATED MICE}

doi:10.1136/gutjnl-2012-302514a.65

${ }^{1}$ G Petts, ${ }^{*}{ }^{2} \mathrm{~A}$ Dhar, ${ }^{1} \mathrm{H}$ Kudo, ${ }^{2} \mathrm{~F}$ Sadiq, ${ }^{3} \mathrm{O}$ Anstee, ${ }^{2} \mathrm{Y}$ Kallis, ${ }^{4} \mathrm{~A}$ Dorling, ${ }^{1} \mathrm{R}$ Goldin, ${ }^{2} \mathrm{M}$ Thursz. ${ }^{1}$ Centre for Pathology, Imperial College, London, UK; ${ }^{2}$ Department of Medicine, Imperial College, London, UK; ${ }^{3}$ Institute of Cellular Medicine, Newcastle University, Newcastle-upon-Tyne, UK; ${ }^{4}$ MRC Centre for Transplantation, King's College, London, UK

Introduction Recent evidence suggests a role for the coagulation cascade in promoting liver fibrosis. However, the cellular basis for this relationship is unclear. In order to explore this relationship we employed two unique transgenic mice strains expressing membrane-tethered tissue factor pathway inhibitor (TFPI) or hirudin (antithrombin) fusion proteins driven by a CD31 promoter. These strains allow for the selective inhibition of tissue factor (TF) or thrombin on endothelial cells, monocytes and platelets expressing CD31.
Aims To evaluate the impact of the targeted inhibition of tissue factor and thrombin on effector cells of liver fibrosis in murine hepatic fibrosis induced by $\mathrm{CCl}_{4}$.

Methods Liver fibrosis was induced in CD31-TFPI, CD31-Hirudin and wild type control mice with 4 weeks carbon tetrachloride $\left(\mathrm{CCl}_{4}\right)$ administered by intraperitoneal injection. Animals were culled and livers extracted for histological and biochemical analysis. Fibrosis was scored using a four point semi-quantitative system and quantified by digital image analysis to determine percentage area of fibrosis. Immunohistochemistry to determine $\alpha$-SMA expression, a marker of hepatic stellate cell activation was performed and the mean number of activated stellate cells was quantified per high power field.

Results The percentage area of fibrosis was significantly less in CD31-TFPI $(1.89 \% \pm 0.26, \mathrm{p}=0.001)$ and CD31-Hirudin mice $(1.04 \% \pm 0.16, p=0.00003)$ in comparison to control mice $(3.66 \% \pm$ 0.39 ). Semiquantitative fibrosis scoring showed a significant difference between the CD31-TFPI (median 2/4) and CD31-Hirudin (median 3/4) mice in comparison with control mice (median $3 / 4$ $\mathrm{p}=0.009$ ) but the difference between the two transgenic strains was not significant. Both transgenic strains demonstrated a significantly reduced mean number of $\alpha$-SMA stellate cells per high power field in comparison to control mice (CD31-TFPI vs control, 7.4 vs 29.4, $\mathrm{p}=0.0002$; CD31-hirudin vs control, 5.25 vs 29.4, $\mathrm{p}=0.0002$ ).

Conclusion CD31 targeted inhibition of TF and thrombin significantly reduces liver fibrosis and stellate cell activation in a murine $\mathrm{CCl}_{4}$ model. The data supports the use of this novel murine model as a tool for investigating the cellular biology of the role of coagulation in liver fibrogenesis. It will also provide information for developing new treatments of human liver fibrosis.

Competing interests None declared.

\section{OC-066 TOLL LIKE RECEPTOR 4 ANTAGONIST PREVENTS ACETAMINOPHEN INDUCED ACUTE LIVER FAILURE IN MICE: A NOVEL THERAPEUTIC STRATEGY}

doi:10.1136/gutjnl-2012-302514a.66

${ }^{1} \mathrm{~N}$ Shah, ${ }^{2} \mathrm{D}$ Dhar, ${ }^{1} \mathrm{M}$ Jover-Cobos, ${ }^{1} \mathrm{~N}$ A Davies, ${ }^{1} \mathrm{R} \mathrm{P}$ Mookerjee, ${ }^{1} \mathrm{R}$ Jalan. ${ }^{1}$ Department of Hepatology, UCL Institute of Hepatology, London, UK; ' Department of Surgery, UCL Institute of Hepatology, London, UK

Introduction Without transplantation, about $40 \%$ of patients with acute liver failure (ALF) die. Its treatment is an unmet need Unregulated inflammation plays an important role in the pathogenesis. Our hypothesis is that Toll like receptor 4 (TLR 4) is critical in the progression of inflammation in ALF. The aims of the study were to determine whether (1) administration of a novel TLR4 antagonist to an acetaminophen (APAP) model of ALF in mice would prevent liver injury. (2) TLR4 antagonist in ALF prolongs survival. (3) TLR4 KO are protected from the liver injury induced by APAP.

Methods Study 1: 3 groups of CD1 mice were studied ( $\mathrm{n}=6$ in each group) Naive, APAP, $500 \mathrm{mg} / \mathrm{kg}$ single dose IP after overnight fasting). APAP+TLR4 antagonist, STM28 (Osaka, Japan); $20 \mu \mathrm{g}$ IP, $1 \mathrm{~h}$ prior to administration of APAP and $6 \mathrm{~h}$ late). Study 2: 3 groups of $\mathrm{C} 57 \mathrm{BL} / 6$ mice were studied $(\mathrm{n}=6)$ in each group. Naive, APAP $(500 \mathrm{mg} / \mathrm{kg}$ single dose IP), APAP+TLR4 antagonist; IAXO (Innaxon) $3 \mathrm{mg} / \mathrm{kg}$ IP, $1 \mathrm{~h}$ prior to administration of APAP and $6 \mathrm{~h}$ later. Study 3: C57BL/6 TLR4 KO were administered APAP $500 \mathrm{mg} /$ $\mathrm{kg}$ and the respective naive controls were administered $500 \mathrm{mg} / \mathrm{kg}$ of saline. Biochemistry was measured using COBAS integra, Cytokines in plasma and tissue homogenate of liver, kidney and brain were measured using ELISA bead array. Brain water was measured using the dry-wet weight method. 